\title{
Seismic vertical array analysis of the velocity structure and phase decomposition during aftershocks of the 2007 Chuetsu-oki earthquake
}

\author{
Kunikazu Yoshida \\ Active Fault Research Center, National Institute of Advanced Industrial Science and Technology, \\ Tsukuba, Ibaraki 305-8567, Japan
}

(Received November 30, 2007; Revised January 21, 2008; Accepted January 30, 2008; Online published November 18, 2008)

\begin{abstract}
A velocity profile derived from $P S$-logging was examined using the mainshock and aftershock records from KK5 and KSH seismic vertical arrays in the Kashiwazaki-Kariwa nuclear power plant, which is located on the focal region. A seismic phase decomposition analysis method was applied to aftershock vertical array records. Peak frequencies of spectral ratios of the aftershock records from the KSH array between various depths were slightly different from those of theoretical transfer functions. A comparison of observed and theoretical $S$-wave travel times at various depths indicated that travel times for the mainshock measured by picking $S$-wave arrival times were identical to the theoretical travel times calculated from the $P S$-logging profile, although travel times for the aftershocks were different from the theoretical travel times. The decomposition analysis of seismic vertical array records from KK5 array for a small aftershock shows that a clear wave packet of surface waves follows just after the direct $S$-wave arrival. In contrast, a decomposition analysis of the KSH array records show that a wave packet of surface waves are not clearly discernable.
\end{abstract}

Key words: The 2007 Chuetsu-oki earthquake, seismic vertical array, site effect, non-linear soil response, seismic phase decomposition.

\section{Introduction}

The 2007 Chuetsu-oki earthquake $\left(M_{\mathrm{JMA}} 6.8\right)$ that occurred at 10:13 on 16 July 2007 (JST) caused strong shaking in Kashiwazaki area. A strong-motion seismograph operating in a free field near the No. 5 plant of the KashiwazakiKariwa nuclear power plant (Tokyo Electric Power Co.) recorded a peak horizontal acceleration of $1223 \mathrm{~cm} / \mathrm{s}^{2}$. Two other seismographs located within $2 \mathrm{~km}$ of the No. 5 plant recorded considerably different waveforms during the mainshock. It is important to try to identify the factors that caused the differences between ground motions at these sites.

Seismic vertical arrays in the power plant recorded ground motions during the mainshock and some aftershocks. Most studies on vertical array records focus on the $S$-wave response based on the spectral ratio of surface to borehole records (e.g., Archuleta and Steidl, 1998). However, a record of ground motion contains both $S$ - and surface waves. It is important to separate $S$-waves from surface waves quantitatively in order to evaluate site effects, for example, an estimation of $S H$-wave soil responses by using observed records.

Yoshida and Sasatani $(2006,2008)$ have proposed a new seismic phase decomposition method using seismic vertical array records. This method decomposes body $(P$ - and

Copyright (c) The Society of Geomagnetism and Earth, Planetary and Space Sciences (SGEPSS); The Seismological Society of Japan; The Volcanological Society of Japan; The Geodetic Society of Japan; The Japanese Society for Planetary Sciences; TERRAPUB.
$S$-waves) and surface wave time histories from vertical array seismograms using transfer functions theoretically calculated from well-investigated velocity structure.

In this article, I analyse the aftershock records from seismic vertical arrays in the power plant, using the seismic phase decomposition method. In particular I pay attention to the validity of the velocity structures before the decomposition analysis.

\section{Site Description and Data Set}

Three vertical arrays are deployed in the KashiwazakiKariwa nuclear power plant, around the focal area of the 2007 Chuetsu-oki earthquake (Fig. 1). Data from two arrays, $\mathrm{KK} 5$ and $\mathrm{KSH}$, were selected in this study because of their high quality. The KK5 array consists of surface and four borehole sensors (Fig. 2). At this array the $S$-wave velocity rapidly increases from $160 \mathrm{~m} / \mathrm{s}$ at the surface to $500 \mathrm{~m} / \mathrm{s}$ at the depth of $36 \mathrm{~m}$ in the Yasuda formation (late Pleistocene), and it gently increases to $870 \mathrm{~m} / \mathrm{s}$ at $312 \mathrm{~m}$ in the Nishiyama (Pliocene to early Pleistocene) and Shiiya (Miocene to Pliocene) formations. The $\mathrm{KSH}$ array is located on a coastal sand dune (Arahama sand dune) that is about $70 \mathrm{~m}$ high, $10 \mathrm{~km}$ long, $2 \mathrm{~km}$ wide and striking about $\mathrm{N} 20^{\circ} \mathrm{E}$. The geologic column at $\mathrm{KSH}$ shows that the Arahama dune sand bed (Holocene, sand deposits in Fig. 2) overlies the Banjin sand bed (late Pleistocene) which in turn overlies the Yasuda formation. $S$-wave velocities of these sand beds remained between $300-350 \mathrm{~m} / \mathrm{s}$ to the depth of $83 \mathrm{~m}$. This array consists of surface and three borehole sensors. 


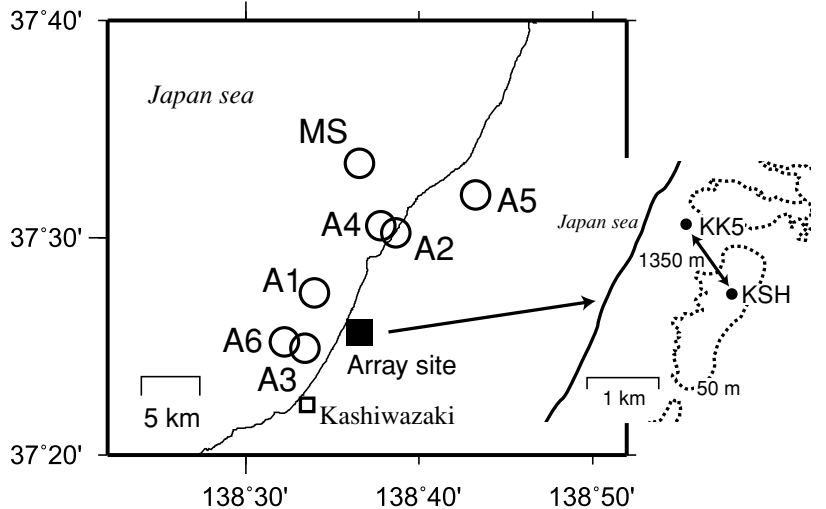

Fig. 1. Map showing the location of the seismic vertical arrays (solid square) and the epicenters of the events (open circles) used in this study. The elevation is shown with 50-m contours.

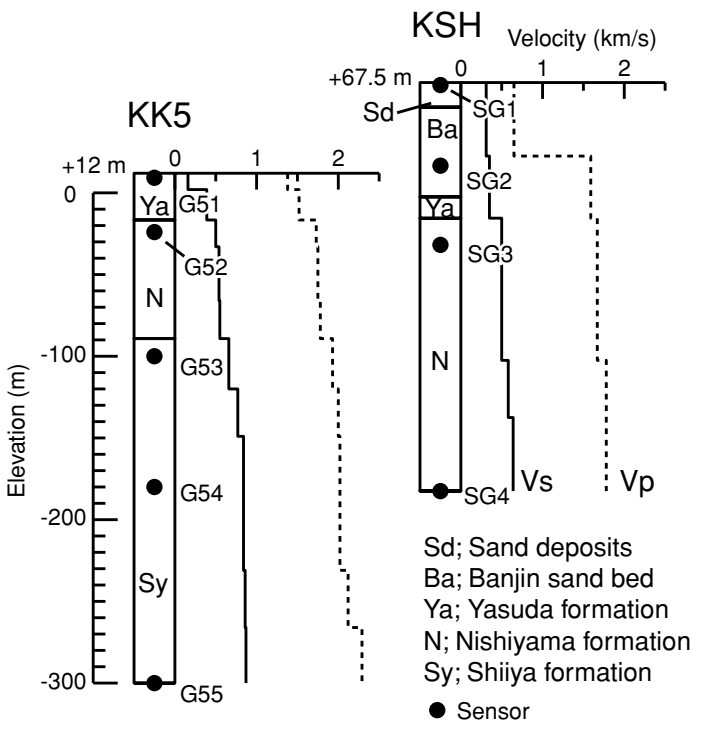

Fig. 2. Array configurations, geologic columns, and velocity structures at the vertical array seismic stations operated by the Tokyo Electric Power.

The mainshock and six aftershocks that were close to the vertical array (epicentral distances $<20 \mathrm{~km}$ ) were recorded (Fig. 1, Table 1). The maximum acceleration during the mainshock is $728 \mathrm{~cm} / \mathrm{s}^{2}$ on the $\mathrm{N} 109^{\circ} \mathrm{E}$ component at SG4, the bottom (250 $\mathrm{m}$ depth) sensor at KSH array. The horizontal components of the records were rotated into $\mathrm{N} 19^{\circ} \mathrm{E}$ and $\mathrm{N} 109^{\circ} \mathrm{E}$, which is along the orientation of the power plant and strike of the sand dune.

\section{Seismic Vertical Array Analysis}

\subsection{Spectral ratios and velocity structures}

Observed spectral ratios between the various depths and theoretical transfer functions for a vertical incident $\mathrm{SH}$ wave were compared. The observed spectra were calculated for each array record using 20 -s, $10 \%$ cosine taper windows beginning $2.0 \mathrm{~s}$ before the $S$-wave arrival. The time window was determined taking into account $S$-wave duration of the mainshock. Spectral ratios were calculated for all combinations of the spectra in each array. Theoretical transfer functions between the surface and various depths were calculated by the propagator matrix method (Aki and Richards,

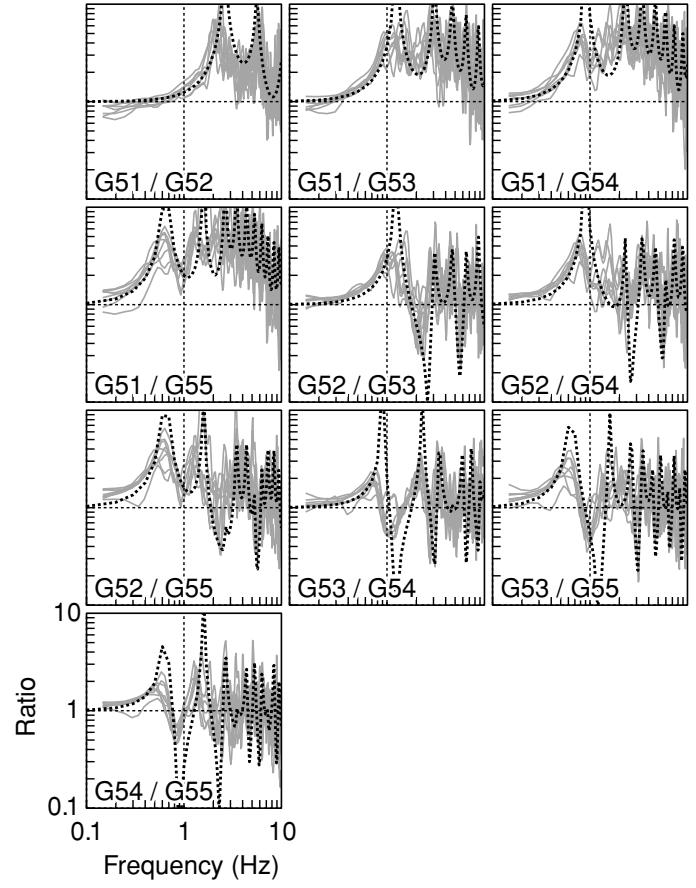

Fig. 3. Comparison of observed spectral ratios between four aftershock records (gray lines) and theoretical transfer functions (dotted lines) at different depths of the KK5 array.

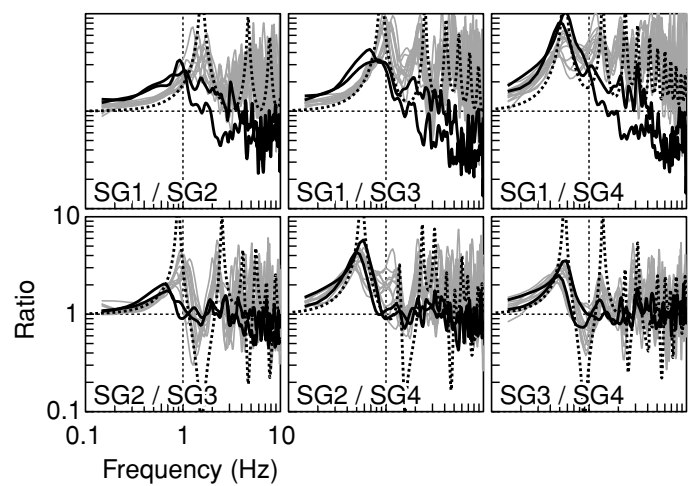

Fig. 4. Comparison of observed spectral ratios between the mainshock records (solid lines), six aftershock records (gray lines), and theoretical transfer functions (dotted lines) at different depths of the KSH array.

1980) for $S$-waves based on the one-dimensional velocity profiles (Fig. 2). $Q_{S}$ ( $S$-wave quality factor) was assumed to be $V_{S} / 15$.

Theoretical transfer functions for the KK5 array agree reasonably well with the observed spectral ratios calculated from KK5 records in $0.1-10 \mathrm{~Hz}$ (Fig. 3). However, the observed spectral ratios vary with the events at frequencies around $1-3 \mathrm{~Hz}$. This feature is clearly seen in the panel of G51/G53 and G52/G54 and suggests a contribution of surface waves.

Observed spectral ratios for aftershock records from $\mathrm{KSH}$ array indicated that the peak frequencies of the observed spectral ratios of the SG1 (surface) record to borehole records are slightly different from the theoretical transfer functions (Fig. 4). In contrast, both the observed spectral ratios and theoretical transfer functions between SG3 and SG4 (SG3/SG4) show good agreement. 
Table 1. Events used in this study (after JMA). Matrix of data is also shown.

\begin{tabular}{cccccccc}
\hline Event & Date, time (JST) & Lat. & Long. & Depth $(\mathrm{km})$ & $M_{\mathrm{j}}$ & KK5 & KSH \\
\hline MS & Jul. 16, 10:13 & 37.557 & 138.609 & 16.8 & 6.8 & - & o \\
A1 & Jul. 16, 11:00 & 37.458 & 138.566 & 22.4 & 3.7 & o & o \\
A2 & Jul. 16, 15:37 & 37.504 & 138.644 & 22.5 & 5.8 & o & o \\
A3 & Jul. 16, 17:42 & 37.415 & 138.557 & 18.9 & 4.2 & o & o \\
A4 & Jul. 16, 21:08 & 37.509 & 138.630 & 20.4 & 4.4 & - & o \\
A5 & Jul. 25, 06:52 & 37.533 & 138.721 & 24.2 & 4.8 & o & o \\
A6 & Aug. 4, 00:16 & 37.420 & 138.537 & 17.9 & 3.2 & - & o \\
\hline
\end{tabular}

Table 2. Observed $P$ - and $S$-wave travel times between array sensors of KK5 array.

\begin{tabular}{c|cccc|cccc}
\hline \multirow{2}{*}{ Event } & $\begin{array}{l}P \text {-wave (s) } \\
\text { G51-G52 }\end{array}$ & G52-G53 & G53-G54 & G54-G55 & G51-G52 & G52-G53 & G53-G54 & G54-G55 \\
\hline Theoretical & 0.022 & 0.043 & 0.040 & 0.057 & 0.108 & 0.138 & 0.105 \\
A1 & 0.04 & 0.04 & 0.03 & 0.06 & 0.11 & 0.14 & 0.11 \\
A2 & 0.02 & 0.05 & 0.04 & 0.05 & 0.15 & 0.13 & 0.10 & 0.13 \\
A3 & 0.02 & 0.05 & 0.03 & 0.06 & 0.11 & 0.14 & 0.12 \\
A5 & 0.02 & 0.05 & 0.05 & 0.05 & 0.10 & 0.13 & 0.10 & 0.13 \\
\hline
\end{tabular}

Table 3. Observed $P$ - and $S$-wave travel times between array sensors of KSH array. $S$-wave arrival time at SG3 for the A2 event could not be picked.

\begin{tabular}{c|ccc|ccc}
\hline \multirow{2}{*}{ Event } & $\begin{array}{l}P \text {-wave (s) } \\
\text { SG1-SG2 }\end{array}$ & SG2-SG3 & SG3-SG4 & $\begin{array}{l}S \text {-wave }(s) \\
\text { SG1-SG2 }\end{array}$ & SG2-SG3 & SG3-SG4 \\
\hline Theoretical & 0.069 & 0.030 & 0.087 & 0.153 & 0.125 & 0.272 \\
MS & 0.09 & 0.03 & 0.09 & 0.16 & 0.12 & 0.28 \\
A1 & 0.09 & 0.04 & 0.09 & 0.21 & 0.14 & 0.28 \\
A2 & 0.09 & 0.06 & 0.08 & 0.19 & - & 0.15 \\
A3 & 0.08 & 0.04 & 0.09 & 0.19 & 0.14 & 0.29 \\
A4 & 0.10 & 0.03 & 0.09 & 0.21 & 0.15 & 0.28 \\
A5 & 0.11 & 0.03 & 0.08 & 0.21 & 0.14 & 0.30 \\
A6 & 0.09 & 0.03 & 0.10 & 0.20 & & \\
\hline
\end{tabular}

The spectral ratios for the mainshock records from KSH array were obviously different from the theoretical transfer function and from the spectral ratios for the aftershocks (Fig. 4). The first peak frequency of the observed spectral ratio of SG1/SG2 was about $0.8 \mathrm{~Hz}$, but the theoretical transfer function predicted a higher frequency of $1.5 \mathrm{~Hz}$. The second and higher peaks of the observed spectral ratio were not clear. In addition, the high-frequency spectral ratios decreased for the mainshock.

In order to examine the validity of the velocity profiles obtained from $P S$-logging, $P$ - and $S$-wave travel times measured by picking $P$ - and $S$-wave arrival times for various depths were compared with theoretical travel times calculated from the velocity profiles (Tables 2 and 3 ). The theoretical travel times between the various depths were calculated by assuming a vertical incidence of the $P$ - and $S$ wave. There is reasonably good agreement between the observed and theoretical travel times at KK5 array. At the $\mathrm{KSH}$ array, the theoretical and observational travel times agree well for the mainshock. For the aftershocks (A1-A6), however, the observed $S$-wave travel times of SG1-SG2 (travel times between SG1 and SG2) and SG2-SG3 (travel times between SG2 and SG3) are larger than the theoretical ones (Table 3). These differences between the observed and theoretical travel times for the aftershocks are one reason for the slight difference in the spectral ratios at KSH be- tween the peak frequencies of the observed spectral ratios for the aftershocks and the theoretical transfer function.

\subsection{Phase decomposition}

In order to examine the wavefield, the seismic phase decomposition method (Yoshida and Sasatani, 2006, 2008) was applied to the vertical array records. I analysed only aftershock records because this method assumes linear soil responses. The $P$ - and $S$-wave velocity structure models at the arrays were made of the $P S$-logging profiles and a deep sedimentary structure model constructed by compiling deep boreholes around the seismic vertical array. The transfer functions between the surface and sensor depths were calculated by the propagator matrix method (Aki and Richards, 1980) for body waves and the compound matrix method (Saito, 1988) for surface waves based on the onedimensional velocity structure models at each array (Fig. 2). The transfer functions for surface waves were calculated by assuming a fundamental mode of Love and Rayleigh waves. Prior to their decomposition, the observed accelerograms were band-pass filtered between $0.5-5 \mathrm{~Hz}$ in order to avoid the instability at the low frequency, and were integrated into velocity time histories.

The result of the vertical array analysis of KK5 array records from the A1 event (Fig. 5) indicates that a wave packet of the $S$-wave arrival (around 8-10 s) consists of a simple $S$-wave pulse and a prominent wave packet of 
a) Observed

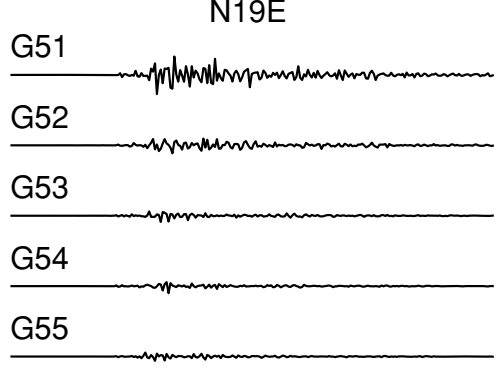

b) Decomposed
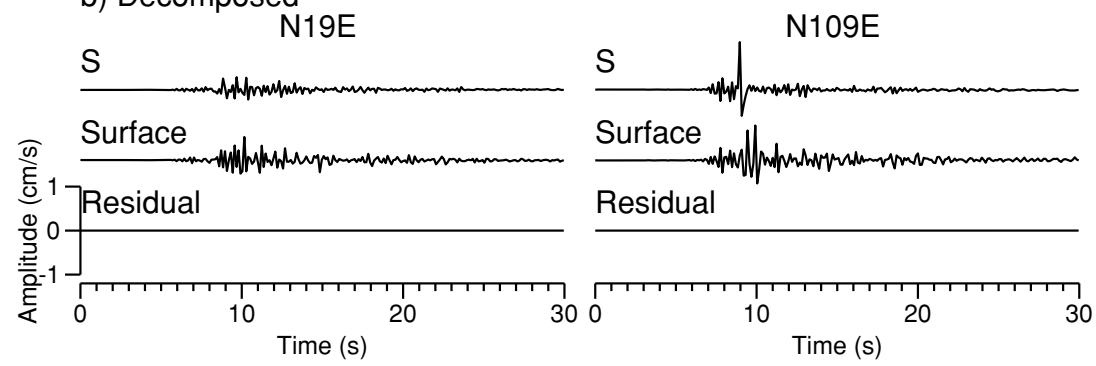

N109E
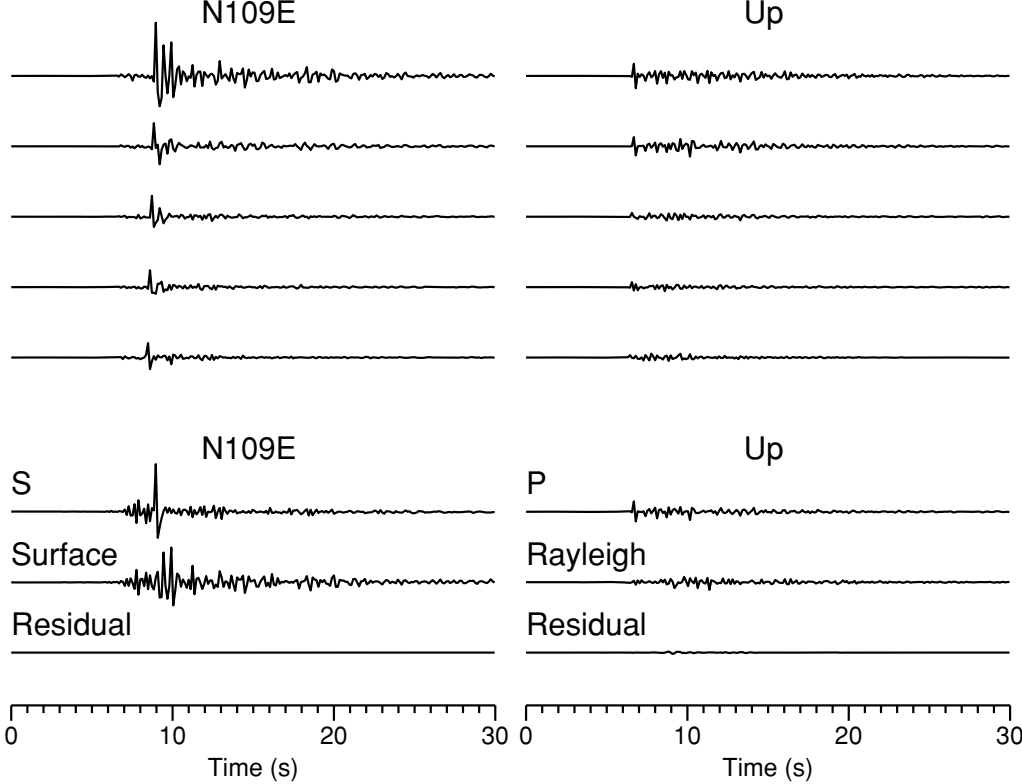

Up

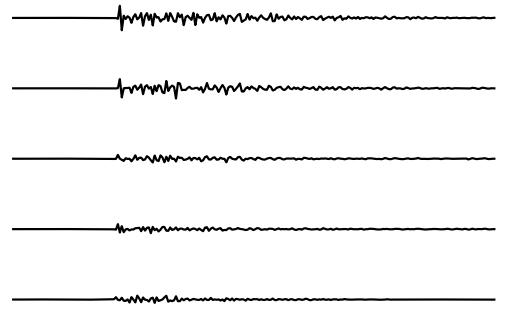

Up

Fig. 5. (a) Vertical array records at KK5 for the A1 event. These are band-pass filtered velocity seismograms: the pass band is from 0.5 to 5.0 Hz. (b) Vertical array analysis results: the decomposed waveforms. The decomposed body $(P$ and $S)$ waves are shown in the top row, the decomposed surface wave terms in the second row, and the residuals in the bottom row.

a) Observed

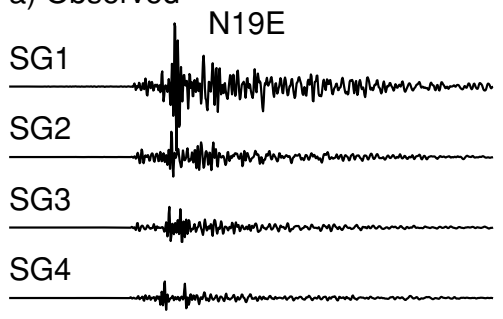

b) Decomposed

N19E

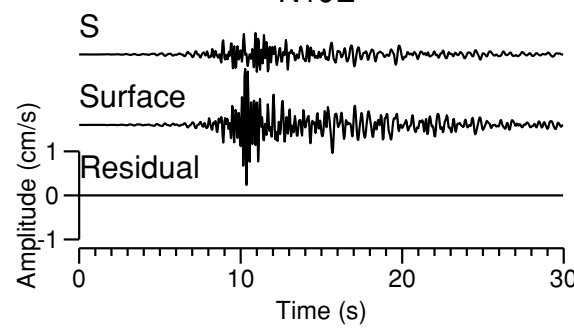

Up

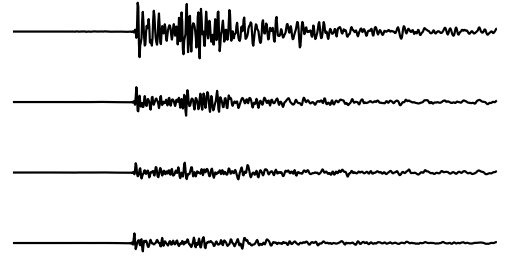

Up
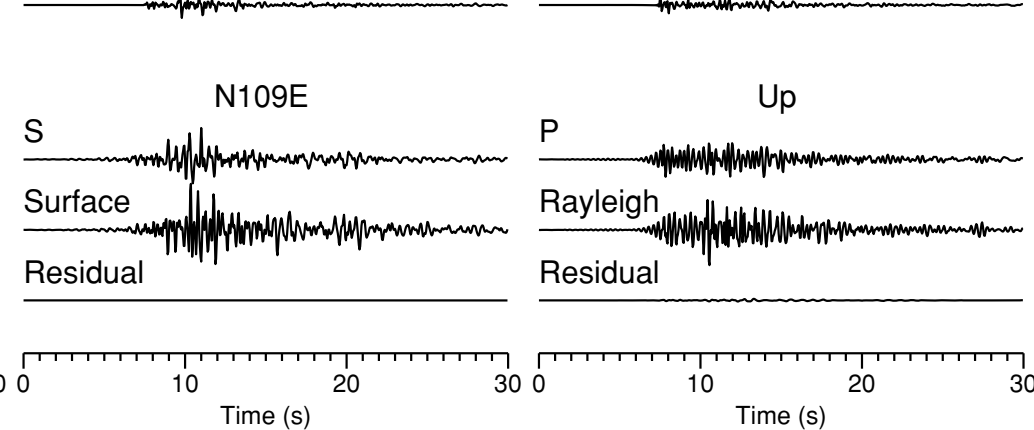

Fig. 6. (a) Vertical array records at $\mathrm{KSH}$ for the A1 event. These are band-pass filtered velocity seismograms: the pass band is from 0.5 to $5.0 \mathrm{~Hz}$. (b) Vertical array analysis results: the decomposed waveforms. The decomposed body $(P$ and $S)$ waves are shown in the top row, the decomposed surface wave terms in the second row, and the residuals in the bottom row. Same format as Fig. 5.

surface waves following the direct $S$-wave, especially in the $\mathrm{N} 109^{\circ} \mathrm{E}$ component. The surface waves with an apparent period of $0.4 \mathrm{~s}(2.5 \mathrm{~Hz})$ have similar amplitude to that of the direct $S$-wave.

Figure 6 shows the decomposition results for the KSH array records. Here, I changed $S$-wave velocities at the first and second layers from 310 and $350 \mathrm{~m} / \mathrm{s}$ to 240 and $340 \mathrm{~m} / \mathrm{s}$, respectively, calibrating with the observed travel times. The result for the A1 event shows that the onset of the $S$-wave is not clear and that the amplitude levels of the surface waves are maintained for more than $15 \mathrm{~s}$. In contrast to the decomposition results for KK5 records, the waveforms of the decomposed $S$-waves are more complicated.

\section{Discussion and Conclusion}

The difference in the observed travel times at the $\mathrm{KSH}$ array between the mainshock and aftershocks suggests that the strong ground motion during the mainshock caused the velocity structure at the KSH array to change. The $S$-wave velocities of the soils at the KSH array before the strong shaking during the mainshock were probably the same as the logging profile. The shift in the peak period of the spectral ratios (Fig. 4) indicates that the surface layers were decreased their rigidity during the mainshock because of 
the non-linear soil response caused by strong shaking. An analysis of array records obtained before the Chuetsu-oki mainshock allows us to clearly observe the change in the velocity structure.

The decomposition analysis of the vertical array records at KK5 from the aftershock reveals that surface wave pulses appeared just after the direct $S$-wave arrival. Surface waves like the A1 event also appeared in a decomposition result of KK5 array records for the A3 event (there is no space to show an analysis result). On the decomposed surface waves at $\mathrm{KSH}$, the surface wave pulses that correspond to the pulses that appeared at KK5 were not found. It is difficult to interpret the analysis results of the $\mathrm{KSH}$ array records in detail due to cross-talk noises between the decomposed body and surface waves. Nevertheless, the differences between the decomposed surface waves from the KK5 and $\mathrm{KSH}$ array suggest that seismic wavefields from Chuetsuoki aftershocks have been complicated.

Acknowledgments. Comments by Haruo Horikawa (AFRC, AIST) were useful. The clarity and completeness of the arti- cle were improved by reviews from Toshimi Satoh and Anatoly Petukhin. The author acknowledges Tokyo Electric Power, Co., for providing the vertical array data. Some of the figures in this paper were produced using GMT (Wessel and Smith, 1998).

\section{References}

Aki, K. and P. G. Richards, Quantitative Seismology-Theory and methods, W. H. Freeman and Company, San Francisco, 1980.

Archuleta, R. J. and J. H. Steidl, ESG studies in the United States: Results from borehole arrays, Proceedings of the Second International Symposium of the Effects of Surface Geology on Seismic Motion, 3-14, 1998.

Saito, M., DISPER80: A subroutine package for the calculation of seismic normal-mode solutions, in Seismological Algorithms, edited by D. J. Doornbos, 293-319, Academic Press, San Diego, 1988.

Wessel, P. and W. H. F. Smith, New, improved version of the Generic Mapping Tools released, EOS, Trans. AGU, 79, 579, 1998.

Yoshida, K. and T. Sasatani, A seismic phase decomposition method using vertical array records, Proceedings of the Third International Symposium on the Effects of Surface Geology on Seismic Motion, 175-184, 2006.

Yoshida, K. and T. Sasatani, Seismic vertical array analysis for phase decomposition, Geophys. J. Int., 174, 707-718, 2008.

K. Yoshida (e-mail: yoshida-ku@aist.go.jp) 